\title{
DISCRETE AND CONTINUOUS ANALYSIS OF POSITRON LIFETIME SPECTRA: A COMPARISON
}

\author{
G. Consolati and F. Quasso
}

Dipartimento di Fisica and Istituto Nazionale per la Fisica della Materia Politecnico di Milano, Piazza Leonardo da Vinci 32, 20133 Milano, Italy

Positron lifetime spectra in poly[1-(trimethylsilyl)]propine membranes were collected at different temperatures and various number of accumulated counts, in order to compare the results from analyses obtained by different decay models: discrete (that is, purely exponential) or continuous (with at least one distribution of lifetimes). Analysis into four discrete components is inadequate, unless a low number of counts ( 1 to 2 millions) is accumulated. Conversely, deconvolutions which allow the ortho-positronium lifetime to be distributed give satisfactory fits, even at the highest statistics (60 millions). An equivalent good fit in terms of $\chi^{2}$ test is obtained with a five discrete components analysis, from which it is possible to extract information on the first moments (centroid and standard deviation) of ortho-positronium lifetime distribution.

PACS numbers: $36.10 . \mathrm{Dr}, 71.60 .+\mathrm{z}, 78.70 . \mathrm{Bj}$

\section{Introduction}

Positron annihilation is a well established technique to study the structure of polymers: the average volume of the nanovoids forming the free volume (in spherical approximation) can be obtained from the measurement of the ortho-positronium (o-Ps) lifetime [1], through deconvolution of an annihilation time spectrum into a discrete number of components. On the other hand, analysis in terms of a distribution of 0 -Ps lifetimes allows one to get the volume distribution function of the nanoholes [2]. In a recent paper [3] we showed the superiority of the latter model with respect to that one where each positron state decays with a single time constant; in the present work we compare the results supplied by two algorithms able to give lifetime distributions, that is, MELT [4] and LT [5]. Furthermore, we show that the main information on such distributions can be obtained through the POSITRONFIT routine [6]. The measurements were carried out on poly[1-(trimethylsilyl)]propine (PTMSP), a membrane which has already been investigated by means of the positron lifetime spectroscopy [7, 8]; it displays unusually long Ps lifetimes. A feature of PTMSP is to be glassy at any temperature, since it decomposes before reaching the glass transition temperature; this makes it suitable to be investigated at different temperatures without interference of microstructural transitions. 


\section{Experimental}

PTMSP membranes were obtained as films (thickness $0.1 \mathrm{~mm}$ ); the sample was formed by placing a sufficient number of films one on another. Details on the membrane preparation can be found elsewhere [9]. The positron source $\left(0.3 \mathrm{MBq}\right.$ of $\left.{ }^{22} \mathrm{Na}\right)$ was embedded into two identical Kapton foils; the sourcesample assembly was inserted into a liquid nitrogen cryostat and positron spectra were obtained under vacuum at six temperatures: $180,240,300,360,420$ and $480 \pm 0.5 \mathrm{~K}$, through a conventional fast-fast coincidence apparatus, having a resolution of about $250 \mathrm{ps}$. For each temperature spectra with increasing statistics were collected, with the maximum number of counts from 5 to $6 \times 10^{7}$. A suitable correction for the positrons annihilated in the Kapton (estimated to be about $12 \%$ ) [10] was taken into account in all the deconvolutions carried out.

\section{Results and discussion}

In Table I the $\chi^{2}$ of the spectra deconvoluted into four discrete components through the POSITRONFIT are reported as a function of the number of counts, for the various investigated temperatures. The $\chi^{2}$ increases with the statistics,

\section{TABLE I}

Results of the $\chi^{2}$ for the deconvolutions through the POSITRONFIT routine into four discrete components, as a function of the accumulated counts (Mc - megacounts).

\begin{tabular}{c|c|c|c|c|c}
\hline \hline$T[\mathrm{~K}]$ & $1 \mathrm{Mc}$ & $5 \mathrm{Mc}$ & $10 \mathrm{Mc}$ & $20 \mathrm{Mc}$ & $50-60 \mathrm{Mc}$ \\
\hline 180 & 1.02 & 1.21 & 1.43 & 1.54 & 3.14 \\
240 & 1.11 & 1.12 & 1.52 & 1.72 & 2.72 \\
300 & 1.03 & 1.11 & 1.42 & 1.78 & 3.19 \\
360 & 1.04 & 1.22 & 1.24 & 1.49 & 2.20 \\
420 & 1.03 & 1.51 & 1.64 & 2.52 & 3.91 \\
480 & 1.02 & 1.31 & 1.42 & 1.89 & 3.24
\end{tabular}

with an almost linear trend, and it generally reaches values higher than 1.5 already below 20 Mcounts. We conclude that the four discrete components model does not satisfactorily describe the annihilation time spectra in PTMSP, unless a low level of accumulated counts is considered. Table II displays the results of the annihilation time spectrum of PTMSP at $300 \mathrm{~K}$ (a statistics of 60 Mcounts was collected), as obtained according to the different algorithms. The data were analyzed into five discrete components by POSITRONFIT, into three discrete components and one lifetime distribution by LT, whilst the number of components cannot be fixed at the input of the MELT routine. The latter supplies a set of possible solutions obtained by varying the entropy weight; the results shown in Table II correspond to a weighted average from the ensemble of solutions. On the other hand, LT allows either a discrete analysis (we verified that the results are identical to those supplied 
TABLE II

Lifetimes $\tau$ [ns] and intensities $I$ [\%] for the time annihilation spectrum of PTMSP at $300 \mathrm{~K}$ and 60 Mcounts. Uncertanties, not shown for brevity, range between 1 and $4 \%$.

\begin{tabular}{l|c|c|c|c|c|c|c|c|c|c|c}
\hline \multicolumn{1}{c|}{ Code } & $\tau_{1}$ & $I_{1}$ & $\tau_{2}$ & $I_{2}$ & $\tau_{3}$ & $I_{3}$ & $\tau_{4}$ & $I_{4}$ & $\tau_{5}$ & $I_{5}$ & $\chi^{2}$ \\
\hline LT & 0.176 & 26.7 & 0.418 & 37.6 & 2.41 & 5.3 & 12.1 & 30.5 & - & - & 1.17 \\
MELT & 0.167 & 23.0 & 0.391 & 40.0 & 2.32 & 6.2 & 8.6 & 11.7 & 14.6 & 18.6 & 1.02 \\
POSITRON- & 0.165 & 24.5 & 0.406 & 39.2 & 2.07 & 4.5 & 7.4 & 10.9 & 14.4 & 20.9 & 1.12 \\
$\quad$ FIT & & & & & & & & & & &
\end{tabular}

by POSITRONFIT), or an analysis where one or more components are distributed according to a Gaussian distribution in the logarithmic scale of lifetimes (i.e., a log-normal distribution). Concerning the first three components, the values of lifetimes and intensities from the different computer codes are in good agreement, and this fact turns out to be true for all the investigated temperatures. The centroid and the standard deviation of the o-Ps lifetime distribution supplied by LT are comparable to the weighted mean and the standard deviation $\sigma$ of the two longest lifetimes from POSITRONFIT

$$
\sigma=\sqrt{\frac{\left(\tau_{4}-\bar{\tau}\right) I_{4}+\left(\tau_{5}-\bar{\tau}\right) I_{5}}{I_{4}+I_{5}}} .
$$

TABLE III

Centroid $\tau$ [ns] and standard deviation $\sigma$ [ns] of $o$-Ps distribution as calculated from the results of the different computer programs.

\begin{tabular}{c|c|c|c|c|c|c|c|c|c}
\hline & \multicolumn{3}{|c|}{ MELT } & \multicolumn{3}{c|}{ LT } & \multicolumn{3}{c}{ POSITRONFIT } \\
\hline$T[\mathrm{~K}]$ & $\tau$ & $\sigma$ & $\chi^{2}$ & $\tau$ & $\sigma$ & $\chi^{2}$ & \multicolumn{1}{c}{$\tau$} & $\sigma$ & $\chi^{2}$ \\
\hline 180 & $12.2(1)$ & $3.4(2)$ & 1.01 & $11.9(1)$ & $4.2(2)$ & 1.14 & $12.2(1)$ & $3.9(2)$ & 1.10 \\
240 & $12.5(1)$ & $3.5(2)$ & 1.06 & $12.5(1)$ & $3.9(2)$ & 1.06 & $12.4(1)$ & $3.7(2)$ & 1.01 \\
300 & $12.3(1)$ & $3.0(2)$ & 1.02 & $12.1(1)$ & $3.4(2)$ & 1.17 & $12.0(1)$ & $3.3(2)$ & 1.12 \\
360 & $12.2(1)$ & $2.3(2)$ & 1.17 & $12.1(1)$ & $2.8(2)$ & 1.22 & $11.8(1)$ & $2.8(2)$ & 1.13 \\
420 & $9.8(1)$ & $2.6(2)$ & 1.02 & $8.9(1)$ & $3.4(2)$ & 1.09 & $9.4(1)$ & $3.0(2)$ & 1.04 \\
480 & $9.4(1)$ & $2.4(2)$ & 0.97 & $9.0(1)$ & $2.9(2)$ & 1.15 & $8.9(1)$ & $2.8(2)$ & 1.08
\end{tabular}

Table III reports the relevant data for the o-Ps distribution of lifetimes at the different temperatures (the decrease in the lifetime at the highest temperatures can be attributed to physical aging of the membrane), from which we can make the following comments:

(a) the routines MELT and LT supply similar results, as far as the trend of the o-Ps distribution of lifetimes is concerned, although deviations up to $27 \%$ for the standard deviation are found. In fact, LT is based - for the sake of simplicity - on a restrictive guess concerning the form of the distribution (log-normal), so different results cannot be surprising.

(b) The standard deviation $\sigma$ of the $o$-Ps lifetimes distribution supplied by MELT is systematically higher than that obtained from LT; on the other hand, 
the values of $\sigma$ obtained from POSITRONFIT through Eq. (1) are intermediate between those supplied by the other codes.

(c) The results obtained from POSITRONFIT are worthwhile, since they show that the main features of the o-Ps lifetime distribution (that is, the first two moments) can be obtained even through a deconvolution into purely discrete components if these latter are suitably interpreted. This program has the advantage to give a single solution, whilst it is known that CONTIN and MELT supply a set of possible solutions, owing to the instability of the inversion problem. It must be pointed out that the average solution supplied by MELT consists of five lifetimes for all the investigated spectra at the highest statistics, although the number of components which can be extracted is always $>6$. This strengthens the results of the deconvolutions carried out through the other two computer codes and eliminates the need to introduce additional distributions, as it is automatically made by CONTIN: as a matter of fact, we generally obtained good $\chi^{2}$ for LT by supposing only the longest component to be distributed.

At last, we observe that the spectra contain a lifetime $\tau_{3}$, which is attributable to o-Ps decay; but it is well separated from the distribution. Work is in progress to understand whether this is a peculiarity of the time annihilation spectrum in PTMSP or it is a feature of Ps decay in polymers, which is generally not revealed due to the much lower number of accumulated counts as well as to the (lower) values of the o-Ps lifetimes.

\section{Acknowledgments}

This work was jointly supported by "Area Materiali" and by "Fondo di Ricerca di Ateneo" of the Politecnico of Milano. Authors are grateful to Professors M. Pegoraro and L. Zanderighi for supplying the samples.

\section{References}

[1] Y.C. Jean, in: Positron Spectroscopy of Solids, Eds. A. Dupasquier, A.P. Mills, IOS Press, Amsterdam 1995, p. 563.

[2] R.B. Gregory, J. Appl. Phys. 70, 4665 (1991).

[3] G. Consolati, R. Rurali, M. Stefanetti, Chem. Phys., to be published.

[4] A. Shukla, M. Peter, L. Hoffmann, Nucl. Instrum. Methods Phys. Res. A 335, 310 (1993).

[5] J. Kansy, Nucl. Instrum. Methods Phys. Res. A 374, 235 (1996).

[6] P. Kirkegaard, N.J. Pedersen, M. Eldrup, in: Positron Annihilation, Eds. L. Dorikens-Vanpraet, M. Dorikens, D. Segers, World Scientific, Singapore 1989, p. 642 .

[7] Yu.P. Yampol'skii, V.P. Shantarovich, F.P. Chernyakovskii, A.I. Kornilov, N.A. Platè, J. Appl. Polym. Sci. 47, 85 (1993).

[8] K. Okamoto, K. Tanaka, M. Ito, H. Kita, Y. Ito, Mater. Sci. Forum 175-178, 743 (1995).

[9] G. Consolati, I: Genco, M. Pegoraro, L. Zanderighi, J. Polym. Sci. B 34, 357 (1996).

[10] K. Plotkowski, T.J. Panek, J. Kansy, Nuovo Cimento D 10, 933 (1988). 